\title{
Does Skin Preparation Influence Surgical Site Infection in Laparotomy Wounds: Comparative Evaluation of Preoperative Skin Preparation With 4\% Chlorhexidine With 5\% Povidone Iodine in Laparotomy Wounds: A Prospective Trial
}

\author{
Ravindran C., Manoj P. Elangovan, Agil B., Reshma A. Cheedhamadathil, Ayana M. Dev, Naveen K. \\ Sreekumar, Babu P. John
}

\begin{abstract}
Introduction: Surgical site infection is a dangerous condition causing a heavy burden on the patient and social health system. Surgical site infections are among the most common hospital acquired infections comprising 14 to $16 \%$ of inpatient infections. There are various factors predisposing the infections and many of them are patient related or disease related. The use of pre-operative skin preparation by effective antiseptic plays an important role in reducing postoperative wound infections. There are several kinds of antiseptics available for preoperative skin preparation; however povidone iodine and spirit are commonly used in clinical practice. Materials and Methods: This study compared the incidence of surgical site infections within $\mathbf{7}$ days of postoperative period in laparotomy wounds prepared using 4\% Chlorhexidine and those prepared with 5\% Povidone iodine for pre-laparotomy skin preparation. Results: This study compared 128, 4\% Chlorhexidine prepared patients and 109, 5\% Povidone iodine prepared patients undergoing various elective $n=114$ and emergency procedures $n=123$. The overall SSI rates in 7 days in the whole group were $\mathbf{1 3 . 4 4}$ $\%$. The SSI rates on the 4\% Chlorhexidine group were $10.16 \%$ and $5 \%$ povidone iodine group were $17.27 \%$ and it was statically significant with a $p$ value of $p=0.00413$ showing significant reduction in the $4 \%$ Chlorhexidine group. The study also compared the SSI rates in elective and emergency procedures in both groups with significant difference in emergency procedures. More variables are also compared between the groups and results were analyzed. Conclusion: This study shows the use of Chlorhexidine $4 \%$ reduces the morbidity of one of the most common wound related complication in laparotomy patients in all categories of laparotomy wounds.
\end{abstract}

Index Terms - SSI, Chlorhexidine 4\%, Povidone iodine 5\%, Laparotomy.

\section{INTRODUCTION}

Surgical Site Infections (SSIs) are infections of tissues, organs, or spaces exposed by surgeons during performance

Published on August 30, 2020.

Ravindran C., Government Medical College, India.

(corresponding e-mail: ravimen ${ }^{\circledR}$ gmail.com)

Manoj P. Elangovan, Government Medical College, India.

Agil B., Government Medical College, India.

Reshma A. Cheedhamadathil, Government Medical College, India.

Ayana M. Dev, Government Medical College, India .

Naveen K. Sreekumar, Government Medical College, India.

Babu P. John, Government Medical College, India. of an invasive procedure [1]. Postoperative SSIs remain a major source of illness and a less frequent cause of death in the surgical patient [2]. These infections number approximately 5,00,000 per year, among an estimated 27 million surgical procedures [3] and account for approximately one quarter of the estimated two million nosocomial infections in the United States each year.

The surgical site infections are the biological summation of multiple compounding factors which includes the inoculum of bacteria introduced into the wound during the procedure, the unique virulence of contaminants, the microenvironment of each wound, and the integrity of the patient's host defense mechanisms. Although an SSI rate of zero may not be achievable, continued progress in understanding the biology of infection at the surgical site and consistent applications of proven methods of prevention will allow furthering reducing the frequency, cost, and morbidity associated with SSI [4].

There are various factors predisposing the infections and many of them are patient related or disease related. The inoculation of bacteria to surgical sites forms one of the most important factors. The use of pre-operative skin preparation by effective antiseptic plays an important role in reducing postoperative wound infections. There are several kinds of antiseptics available for preoperative skin preparation; however povidone iodine and $4 \%$ chlorhexidine are commonly used in clinical practice [5]. 4\% chlorhexidine and $5 \%$ povidone iodine are proven to be effective against most skin commensals [6].

The present study was undertaken to compare and evaluate the efficacy of chlorhexidine versus povidone iodine in abdominal surgeries in preventing SSIs.

This study compared 128, 4\% Chlorhexidine prepared patients and 110, 5\% Chlorhexidine prepared patients undergoing various elective $\mathrm{n}=111$ and emergency procedures $n=127$ and compared the incidence of surgical site infections within 7 days of postoperative period in laparotomy wounds prepared using 4\% Chlorhexidine and those prepared with 5\% Povidone iodine for pre-laparotomy skin preparation. The study also compared the SSI rates in elective and emergency procedures and more variables are also compared between the groups and results were analyzed. 


\section{MAterial And MethodS}

We conducted a prospective study of 237 patientswho underwent elective and emergency laparotomies in the Department of General Surgery, Government Medical College, Thrissur.

A prospective Cross-sectional study design was undertaken in patients undergoing laparotomy procedures where those prepared with $4 \%$ Chlorhexidine were grouped as group 1 and 5\% Povidone iodine as group 2. The subjects were matched for factors affecting wound infection like age, diabetes, and nutritional status, anemia as far as possible in elective and emergency patients.

The study recorded the date and time of preparation, appearance, sloughing tissue or exudates, and skin wound swabs taken on post-operative days if any signs of clinical surgical site infection were noted.

Following laparotomy and completion of procedure, Linea Alba was closed with synthetic absorbable Loop PDS No.1 suture. In all cases the wound was wiped with sterile gauze and to wash the surgical wounds Normal Saline Lukewarm was preoperatively prepared in a sterile setting. The wound was dried with sterile gauze and closed with Skin staplers or with 2.0Ethilon sutures.

Post operatively wounds were observed daily for signs of wound infection like discharge, local induration, redness, local rise of temperature for a period of one week postoperatively by the junior residents and the principal investigator. Post-operative wound swabs were taken and the culture status was recorded when there was evidence of wound infection by the corresponding Units. Surgical site infection rates were analyzed statistically in matched groups and compared. Patient confidentiality was always maintained.

Data collected from everyone were collated and quantitative data was analyzed using mean, standard deviation \& appropriate statistical test of significance.

\section{RESUlts AND ANALYSIS}

We analyzed 237 cases of laparotomy wounds which were included in the study. The data collected was entered in Microsoft Excel and analyzed using SPSS version 16.

The presence of surgical site infection in pulse irrigation and standard irrigation was associated with the socio demographic variables.

The distribution of cases showed an equitable distribution and almost matched for the variables of the Age, sex. Age groups, co morbidities (Fig. 1 and 2).

There were 114 Elective cases and 123 emergency cases spread over a variety of conditions as depicted in Table I (a) and Table I (b). There was no statistical significance due to difference in age in the two groups undergoing elective and emergency surgeries. Majority of the procedures performed in elective cases were clean $24.7 \%$ or clean contaminated $49.54 \%$ while a major proportion of procedures in emergencies were due to contaminated cases $58.33 \%$ Table II.

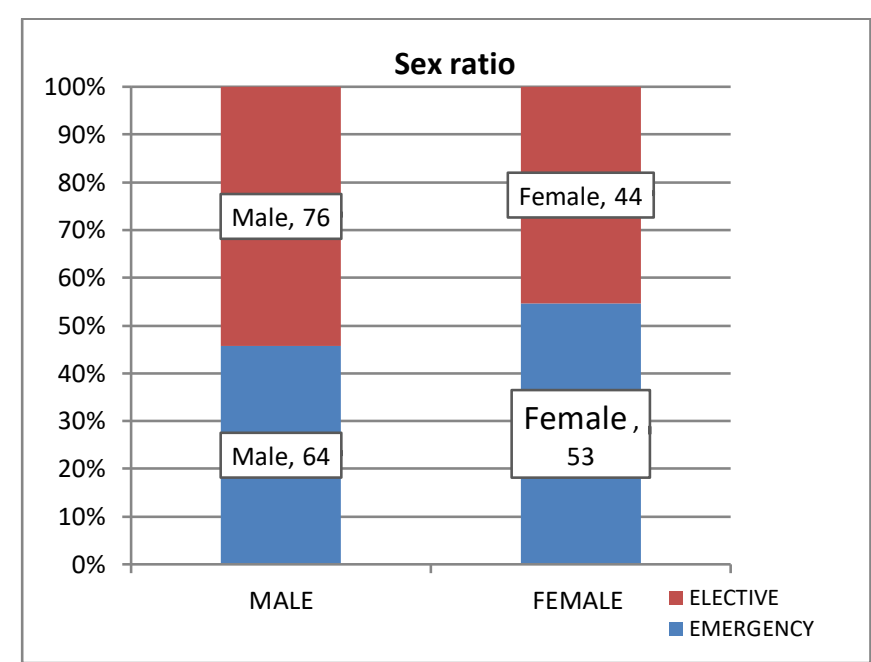

Fig. 1. Sex distribution of cases were statistically insignificant $\mathrm{p}$ Value $>0.005$

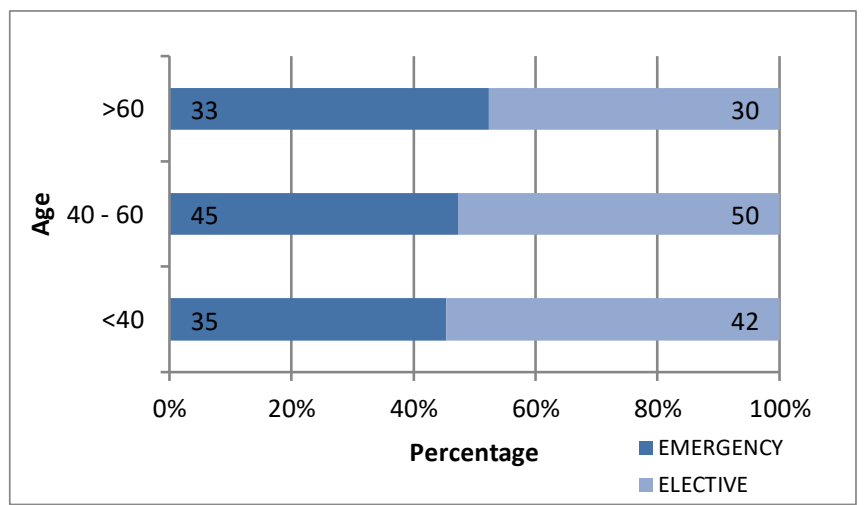

Fig. 2. Common Age group in both Elective and emergencies were 40-60 years.

\begin{tabular}{lc}
\multicolumn{2}{c}{ TABLE I(A): EMERGENCY PROCEDURES } \\
\hline & N = 128 \\
\hline Condition & Number \\
\hline Blunt trauma abdomen & 18 \\
Emergency cholecystectomy & 6 \\
DU perforation & 33 \\
Pre pyloric ulcer perforation & 18 \\
Appendicular perforation & 14 \\
Appendicular mass & 6 \\
Mekels diverticulitis & 4 \\
Intestinal obstruction & 16 \\
Colonic perforation & 4 \\
Splenic abscess & 2 \\
Panceatic necrosis & 2 \\
Complicated internal hernia & 1 \\
Irreducible incisional hernia & 4 \\
\hline
\end{tabular}

TABLE I(B): ELECTIVE PROCEDURES

\begin{tabular}{lc}
\hline Condition & Number \\
\hline Fundoplication & 2 \\
Hellers myotomy & 2 \\
CA stomach & 24 \\
CA pancreas & 6 \\
Gist & 6 \\
RT colon cancer & 12 \\
GB cancer & 1 \\
CBD exploration & 2 \\
Left hepatectomy & 2 \\
Splenectomy & 2 \\
Carcinoma rectum & 22 \\
Small bowel tumours & 3 \\
Mescebtric cyst & 3 \\
Carcimona ovary & 12 \\
CA endometrium & 8 \\
Retroperitoneal tumour & 4 \\
Retroepritoneal abscess & 1 \\
\hline
\end{tabular}


TABLE II: TYPE OF SURGERIES

\begin{tabular}{ccc}
\hline Particulars & Emergency & Elective \\
\hline CLEAN SURGERIES & $5(4.17 \%)$ & $27(24.77 \%)$ \\
CLEAN CONTAMINATED & $48(37.5 \%)$ & $54(49.54 \%)$ \\
CONTAMINATED & $75(58.33 \%)$ & $28(25.69 \%)$ \\
\hline
\end{tabular}

Type of anesthesia also followed a standard pattern both in elective and emergency procedures.

The overall SSI rates in 7 days in the whole group were $32 / 238(10.37 \%)$. The SSI rates on the $4 \%$ chlorhexidine group was 13/128 (10.16\%) and Standard irrigation group was $18 / 109$

$(16.56 \%)$ (Table III (a)). When all procedures were combined and assessed for the surgical site infection there is a significant difference between $4 \%$ chlorhexidine and $5 \%$ povidone iodine procedure with a $p$ value of $<0.05$ ( $p$ value $=0.004133071)$ suggesting that there is a significant reduction in SSI in the $4 \%$ chlorhexidine group after all laparotomies (Table III (a)).
In elective procedures the incidence of the surgical site infection was $11.4 \%$ and there was a significant difference between the $4 \%$ chlorhexidine group $8.77 \%$ and 5\% povidone iodine group $14.2 \%(\mathrm{p}$ value $=0.002)($ Table 3 (b)).

In emergency procedures the incidence of the surgical site infection was $16.6 \%$ and there was a significant difference between the $4 \%$ chlorhexidine $11.26 \%$ and $5 \%$ povidone iodine $19.23 \%$ group ( $\mathrm{p}$ value $=0.002$ ). SSI is significantly less when $4 \%$ chlorhexidine is employed for emergency surgeries (Table III (b)).

We also analyzed the incidence of infection in elective cases in pulse irrigation and standard irrigation techniques and found that the incidence of infection doesn't have any correlation between the groups $(\mathrm{p}$ value $=0.668235142)$ (Table III (b)).

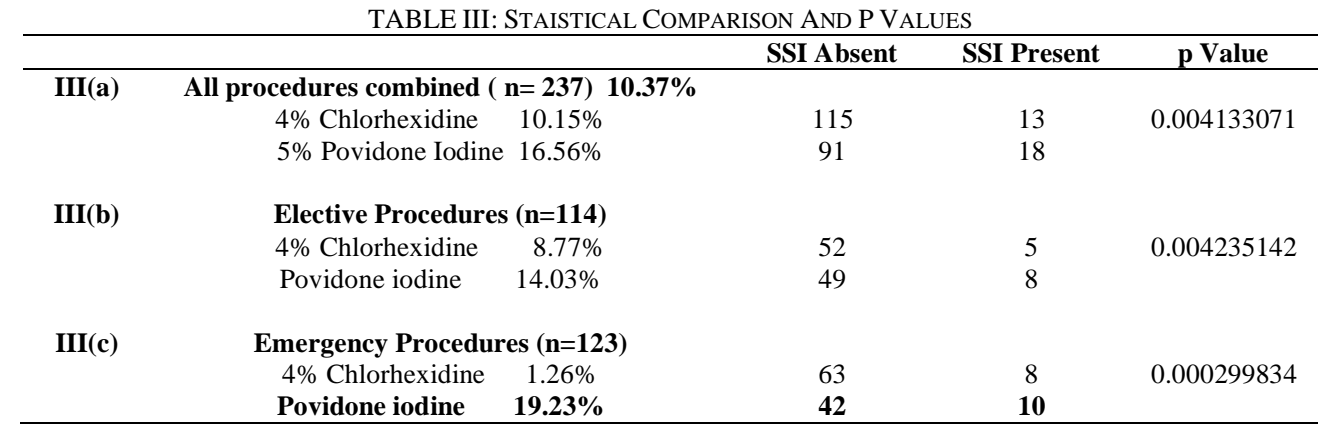

There was no difference in the type of surgeries, the bacterial inoculums and type of anesthesia used for the procedures. This suggested that there is a statistically significant reduction in the $4 \%$ Chlorhexidine group in all laparotomies and Emergency procedures.

\section{Discussion}

Surgical site infections cause heavy burden on the patient and social health system. Such infections lengthen hospital stay for an average of seven days and results in higher costs. Surgical site infections are sub classified to Superficial incisional SSI, Deep incisional SSI and Organ/Space SSI.

Surgical site infection (SSI) is a dreaded postoperative complication that affects approximately $5 \%$ of all patients undergoing surgery [7]. It is associated with prolonged length of hospital stay, prolonged postoperative recovery time, higher hospital readmission rates, and higher morbidity and mortality rates than patients without SSI [8]. The majority of SSIs are caused by contamination of a surgical incision with bacteria from the patient's own body [9]. There are several antiseptic skin cleansing agents available to the surgeon to use for patients undergoing clean, clean-contaminated, contaminated, and dirty surgery [10]. The traditional antiseptic cleansing agent of choice is povidone iodine (PI). It is cheap, effective, and the most commonly used agent of choice worldwide [11]. Chlorhexidine-alcohol is a newer skin preparation agent, commonly composed of $2 \%$ chlorhexidine gluconate and $70 \%$ isopropyl alcohol [12]. Although more expensive than PI, it represents an alternative skin antiseptic agent and is reported to have a more rapid onset of action than PI and has persistent activity in the presence of body fluids [13].

Chlorhexidine is probably the most widely used biocide and is a cheap, easy to use bactericidal. Povidone iodine functions by release of elemental iodine which binds irreversibly with protein. Although less reactive than chlorine, iodine is rapidly bactericidal, fungicidal, tuberculocidal, viricidal, and sporicidal [14].

Despite increasing evidence for newer skin antiseptic cleansing agents, there is still clinical equipoise concerning which agent is associated with a lower risk of postoperative surgical site infections.

The overall SSI rates in 7 days in the whole group were (10.37\%). The SSI rates on the $4 \%$ chlorhexidine group was $10.16 \%$ and Standard irrigation group was $16.56 \%$ (Table $3 a)$.When all procedures were combined and assessed for the surgical site infection there was a significant difference between $4 \%$ chlorhexidine and 5\% povidone iodine procedure with a $\mathrm{p}$ value of $<0.05(\mathrm{p}$ value $=0.004133071)$ suggesting that there is a significant reduction in SSI in the $4 \%$ chlorhexidine group after all laparotomies (Table III (a)). Primary meta-analysis including six prospective randomized trials have pointed out that $4 \%$ chlorhexidine alcohol was associated with lower rates of postoperative SSIs in patients undergoing clean and clean-contaminated surgery.

In elective procedures the incidence of the surgical site infection was $11.4 \%$ and there was a significant difference between the 4\% chlorhexidine group $8.77 \%$ and 5\% povidone iodine group $14.2 \%(\mathrm{p}$ value $=0.002)($ Table III 
(b)) and this has been proved by various similar studies on elective cases.

In emergency procedures the incidence of the surgical site infection was $16.6 \%$ and there was a significant difference between the $4 \%$ chlorhexidine $11.26 \%$ and $5 \%$ povidone iodine $19.23 \%$ group $(\mathrm{p}$ value $=0.002$ ). SSI is significantly less when $4 \%$ chlorhexidine is employed for emergency surgeries (Table III (c)).

Chlorhexidine is associated with a lower rate of SSI in clean and clean-contaminated surgery and is also documented in various studies. That is substantiated by our study and Maiwald and Chan in 2012 [15].

Several studies have shown the advantages of chlorhexidine in pre-operative preparations but so far, no studies have been done to compare its efficacy versus povidone iodine for contaminated and clean-contaminated surgeries in India.

\section{CONCLUSION}

The goal of the study was to assess whether $4 \%$ Chlorhexidine or Povidone iodine was effective in reducing the surgical site infections of laparotomy wounds. The study results suggest that there is a decrease in the incidence of SSI in $4 \%$ chlorhexidine group compared to 5\% Povidone iodine and also it decreases the postoperative stay, morbidity and cost in both emergency and elective laparotomy patients. More and more standardisation of the groups in terms of types of procedures, co-morbid factors and compounding factors should be considered in further studies for gathering more information on the impact of this technique. Despite increasing evidence for newer skin antiseptic cleansing agents, there is still clinical equipoise concerning which agent is associated with a lower risk of postoperative SSI.

\section{REFERENCES}

[1] CF Brunicardi, DK Andersen, Schwartz's manual of surgery. 9th ed. New York, NY: McGraw-Hill; 2010.

[2] RL. Nichols, "Postoperative infections in the age of drug-resistant gram-positive bacteria," Am J Med, Vol.104, pp.11S-16S., 1998.

[3] Centers for Disease Control and Prevention, National Center for Health Statistics Vital and Health Statistics, Detailed diagnoses and procedures national hospital discharge survey 1994. Hyattsville (MD): Department of Health and Human Services; vol. 127, 1997.

[4] G Fabiano, A Pezzolla, MA Filograna, F Ferrarese, "Risk factors of surgical wound infection," Ann Ital Chir, Vol.175 No.1, pp.11-6, 2004.

[5] V Paocharoen, C Mingmalairak, A Apisarnthanarak, "Comparison of surgical wound infection after preoperative skin preparation with $4 \%$ chlorhexidine [correction of chlorhexidine] and povidone iodine: a prospective randomized trial," J Med Assoc Thai. Vol.92, No. 7, pp. 898-902, 2009.

[6] A Guzel, T Ozekinci, U Ozkan, Y Celik, A Ceviz, D Belen, "Evaluation of the skin flora after chlorhexidine and povidone-iodine preparation in neurosurgical practice" Surg Neurol, Vol.71 No. 2, pp.207-10, 2009.

[7] AJ Mangram, TC Horan, ML Peearson, LC Silver, WR Jarvis, "Guidelines for prevention of surgical site infections. Hospital Infection Control Practices Advisory Committee," Infect Control Hosp Epidemiol, Vol. 20, pp.250-278, 1999.

[8] GW Denton. Chlorhexidine. In: Block SS, ed. Disinfection, sterilization, and preservation. 5th ed. Philadelphia: Lippincott Williams \& Wilkins, 2001, pp. 321-36.

[9] PJ Culligan, K Kubik, M Murphy, L Blackwell, J Snyder, "A randomized trial that compared povidone iodine and chlorhexidine as antiseptics for vaginal hysterectomy," Am J Obstet Gynecol, Vol.192, pp.422-425, 2005.
[10] RV Ostrander, MJ Botte, ME Brage, "Efficacy of surgical preparation solutions in foot and ankle surgery," J Bone Joint Surg Am, Vol.87, pp.980-985, 2005.

[11] Bibbo C, Patel DV, Gehrmann RM, Lin SS. "Chlorhexidine provides superior skin decontamination in foot and ankle surgery: a prospective randomized study," Clin Orthop Relat Res, Vol. 438, pp.204-208, 2005 .

[12] AM Milstone, CL Passaretti, TM Perl, "Chlorhexidine: Expanding the armamentarium for infection control and prevention," Clin Infect Dis, Vol.46, pp.274-81, 2008

[13] Hebl JR, Niesen AD. "Infectious complications of regional anesthesia," Curr Opin Anaesthesiol, Vol.24, pp.573-80, 2011.

[14] JC Dumville, E McFarlane, P Edwards, A Lipp, A Holmes, "Preoperative skin antiseptics for preventing surgical wound infections after clean surgery," Cochrane Database Syst Rev, No.3, CD003949, 2013.

[15] M Maiwald, ESY Chan. "The forgotten role of alcohol: a systematic review and meta-analysis of the clinical efficacy and perceived role of chlorhexidine in skin antisepsis," PLoS ONE, Vol.7, No. 9, 2012. 